REPRESENTATION THEORY

An Electronic Journal of the American Mathematical Society

Volume 10, Page 48 (January 11, 2006)

S 1088-4165(06)00304-9

\title{
ERRATUM: EXPLICIT MATRICES FOR IRREDUCIBLE REPRESENTATIONS OF WEYL GROUPS
}

JOHN R. STEMBRIDGE

In the paragraph preceding Lemma 2.4 of [1], we defined an ordering $s_{1}, \ldots, s_{n}$ of the simple reflections to be graceful if for all $i<j<k$, we have $i$ and $k$ adjacent in the Coxeter graph (i.e., $s_{i}$ and $s_{k}$ do not commute) only if $j$ and $k$ are also adjacent. We also wrote that it is "easy to check that the standard order we have chosen for each Weyl group is graceful." However, this is not correct for the orderings we used for the Weyl groups of type $E$; in fact, the triple $(i, j, k)=(1,2,3)$ clearly violates the condition.

One way to fix this error is simply to interchange $s_{2}$ and $s_{3}$ in the standard ordering. The remainder of the paper remains completely unaffected; however, this does change the matrices that our algorithm would construct.

A better way to fix the error is to improve the definition. We should instead define the ordering $s_{1}, \ldots, s_{n}$ to be graceful if for all $i<j$ such that $i$ and $j$ are adjacent in the Coxeter graph, each of $s_{i}, s_{i+1}, \ldots, s_{j-1}$ belong to distinct irreducible components of the parabolic subgroup generated by $s_{1}, s_{2}, \ldots, s_{j-1}$.

The orderings we used for the simple reflections in the Weyl groups of type $E$ do satisfy this weaker condition, Lemma 2.4 remains valid as stated, and the first paragraph of the proof now simplifies to the following:

"For clarity, we will assume that $r=n-3$; the general case follows by essentially the same argument. Given that the ordering is graceful, it follows that $W_{n-1}$ is a direct product of parabolic subgroups, say $W_{I} \times W_{J}$, with generating sets $I$ and $J$ that include $s_{n-1}$ and $s_{n-2}$, respectively."

No other changes are needed. In particular, the statements and proofs of Proposition 2.5 and Corollary 2.6 are valid in their original forms.

\section{REFERENCES}

[1] J. R. Stembridge, Explicit matrices for irreducible representations of Weyl groups, Represent. Theory 8 (2004), 267-289. MR2077483 (2005h:20096)

Department of Mathematics, University of Michigan, Ann Arbor, Mi 48109-1043

E-mail address: jrs@umich.edu

Received by the editors December 6, 2005

2000 Mathematics Subject Classification. Primary 20F55, 20C40; Secondary 05E15, 20-04.

(C)2006 American Mathematical Society 